\title{
The absolute motion of the peculiar cluster NGC $6791^{\star}$
}

\author{
L. R. Bedin ${ }^{1}$, G. Piotto ${ }^{2}$, G. Carraro ${ }^{2,3}$, I. R. King ${ }^{4}$, and J. Anderson ${ }^{5}$ \\ ${ }^{1}$ European Southern Observatory, Karl-Schwarzschild-Str. 2, 85748 Garching, Germany \\ e-mail: 1bedin@eso.org \\ 2 Dip. di Astronomia, Univ. degli studi di Padova, vic. Osservatorio 2, 35122 Padova, Italy \\ e-mail: piotto@pd.astro.it \\ 3 Andes Fellow, Departamento de Astronómia, Universidad de Chile, Casilla 36-d, Santiago, Chile \\ e-mail: gcarraro@das.uchile.cl \\ 4 Dept. of Astronomy, Univ. of Washington, Box 351580, Seattle, WA 98195-1580, USA \\ e-mail: king@astro.washington.edu \\ 5 Dept. of Physics and Astronomy, Mail Stop 108, Rice University, 6100 Main Street, Houston, TX 77005, USA \\ e-mail: jay@eeyore.rice.edu
}

Received 21 July 2006 / Accepted 17 October 2006

\section{ABSTRACT}

\begin{abstract}
We present improved values of the three components of the absolute space velocity of the open cluster NGC 6791. One HST ACS/WFC field with two-epoch observations provides astrometric measurements of objects in a field containing the cluster center. Identification of 60 background galaxies with sharp nuclei allows us to determine an absolute reference point, and measure the absolute proper motion of the cluster. We find $\left(\mu_{\alpha} \cos \delta, \mu_{\delta}\right)_{\mathrm{J} 2000.0}=(-0.57 \pm 0.13,-2.45 \pm 0.12) \mathrm{mas} \mathrm{yr}^{-1}$, and adopt $V_{\mathrm{rad}}=-47.1 \pm 0.7 \mathrm{~km} \mathrm{~s}^{-1}$ from the average of the published values. Assuming a Galactic potential, we calculate the Galactic orbit of the cluster for various assumed distances, and briefly discuss the implications on the nature and the origin of this peculiar cluster.
\end{abstract}

Key words. astrometry - open clusters and associations: individual: NGC 6791 - Galaxy: kinematics and dynamics

\section{Introduction}

NGC 6791 is a unique object in our Galaxy. Usually classified as an open cluster, it has a number of peculiarities which leave its origin and its nature quite enigmatic. It is more massive (at least $\left.4000 m_{\odot}\right)$, more metal-rich $([\mathrm{Fe} / \mathrm{H}] \sim+0.4$, Carraro et al. 2006; Gratton et al. 2006), and older ( $\approx 9$ Gyr, King et al. 2005) than most known open clusters. In contrast with other open clusters, which lie close to the Galactic plane, its distance of $4000 \mathrm{pc}$ (King et al. 2005) and Galactic latitude of $11^{\circ}$ put NGC $6791 \sim 1 \mathrm{kpc}$ above the plane. The cluster is also anomalous with respect to the radial abundance gradient and the age-metallicity relation of the Galactic disk. (See discussion in Carraro et al. 2006.)

All of these peculiarities make NGC 6791 both an interesting and a challenging object, and they stimulated us to undertake a study of it with deep HST imaging. In such HST programs we have, whenever possible, included a second epoch of observation, in order to use proper motions to separate cluster stars from the field. The second epoch also allows us, here, to study the motion of the cluster itself.

We have already published two papers based on the firstepoch observations alone. The first of them reported the discovery (Bedin et al. 2005) of an anomalous white dwarf cooling sequence. The second paper (King et al. 2005) was devoted to

* Based on observations with the NASA/ESA Hubble Space Telescope, obtained at the Space Telescope Science Institute, which is operated by AURA, Inc., under NASA contract NAS 5-26555, under programs GO-9815, and GO-10471. the main sequence, and included a preliminary mass function, which we found to be rather flat.

Along with our proper motion, the availability in the literature of radial velocities for a number of cluster members allows us to determine all three components of the absolute motion of NGC 6791 and to infer some properties of its orbit, which will shed some light on the possible origin of this object. A detailed study of the cluster main sequence (down to the hydrogenburning limit) and of the white dwarf cooling sequence will be presented in forthcoming papers.

\section{Observations, data reduction, and proper motions}

All of the observations used for the proper-motion measurement come from our HST programs GO-9815 and GO-10471 (PI King), which were separated by $\sim 2$ years. For precise astrometric measurements and a more accurate assessment of the errors, we had taken particular care to dither our images properly, with both whole-pixel and fractional-pixel offsets (following the general recipes given in Anderson \& King 2000). Table 1 describes the ACS/WFC observations used in this work. The first of the two programs also included images in the $F 606 \mathrm{~W}$ band, but we used only $F 814 \mathrm{~W}$ images for our proper motions, in order to avoid any possible filter-dependent systematic errors.

We measured positions and fluxes for every star in every F814W_FLT exposure, using library effective PSFs and the software programs documented in Anderson \& King (2006). We then generated a master list of all the stars, and collated all the observations of each star. As in Bedin et al. (2003), we 
Table 1. Data set used in this work.

\begin{tabular}{cccc}
\hline \hline Epoch(date) & Exp. time & Filt & Go \\
\hline I (17 July 2003) & $2 \times 1142 \mathrm{~s}+4 \times 1185 \mathrm{~s}$ & $F 814 W$ & 9815 \\
II (13 July 2005) & $2 \times 1200 \mathrm{~s}+4 \times 1264 \mathrm{~s}$ & $F 814 W$ & 10471 \\
\hline
\end{tabular}

used the best distortion corrections available (Anderson 2002, 2005) to correct the raw positions that we had measured from the _FLT exposures.

We carefully constructed the reference frame, as follows. We measured a simple centroid position for each bright star in the first_DRZ image, and found by least squares a linear transformation between those positions and the positions of the same stars in the corresponding _FLT image. We then rotated the frame of the _FLT image so that the $y$ axis points exactly north, and rescaled it to agree with the pixel size of the _DRZ image, which is $50 \mathrm{mas} /$ pixel. This is our reference frame. From the header of the _DRZ image we got the RA and Dec of the point in our reference frame that corresponds to the position given in the header; this allows us to combine the internal accuracy of our PSF-measured positions with the absolute orientation and scale information from calibrated pipeline products.

As reference stars we identified cluster members in the color-magnitude diagram, and used only those stars for the transformation from each exposure into the reference frame. (See Bedin et al. 2003, for details.) We thus ensured that the proper motions are measured relative to the bulk cluster motion. Carraro et al. (2006) give an estimate of $2.2 \pm 0.4 \mathrm{~km} \mathrm{~s}^{-1}$ for the internal dispersion of radial velocities; for a distance of $4 \mathrm{kpc}$,

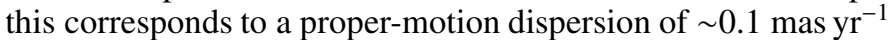
( $~ 0.005$ ACS/WFC pixel in two years). This means that the relative motions of the cluster stars should all be zero to within the measurement errors. We iteratively removed from the member list some stars that had field-star-type motions, even though their colors placed them near the fiducial cluster sequence. Field-star proper motions will be discussed very briefly at the end of this section.

Finally, in order to minimize the influence of any uncorrected distortion on transformations into the reference frame, we used for each object a local transformation based on the nearest $\sim 50$ well-measured cluster stars. With all these precautions, we found that for stars with $>500 \mathrm{DN}$ in their brightest pixel we could measure positions in a single image with an error $<0.05$ pixel in each coordinate. We note that the relatively high background in our images $(\sim 65 \mathrm{DN})$ makes the astrometric effect of charge-transfer efficiency negligible, and also that the red-halo effect that disturbs ACS/HRC images is negligible in WFC images taken with the $F 814 W$ filter.

A visual inspection of the images reveals many background galaxies. Since 60 of these show a point-like nucleus, we used our ePSF-fitting procedure to measure positions for them also. We transformed the position of each galaxy into the reference frame using the same kind of transformations that we used for the stars. With $N$ observations for each galaxy at each epoch, we calculated the random error for each galaxy as $\sigma / \sqrt{N-1}$, where $\sigma$ comes from the agreement among the independent measurements. We also added in quadrature the error in the transformation, based on the residuals of the stars used to compute the transformation. As expected, the errors in multiple measurements of galaxy positions are several times as large as typical errors in star positions, and depend strongly on the galaxy morphology. Nevertheless, on average these galaxies provide a good reference frame for the measurement of absolute proper motions

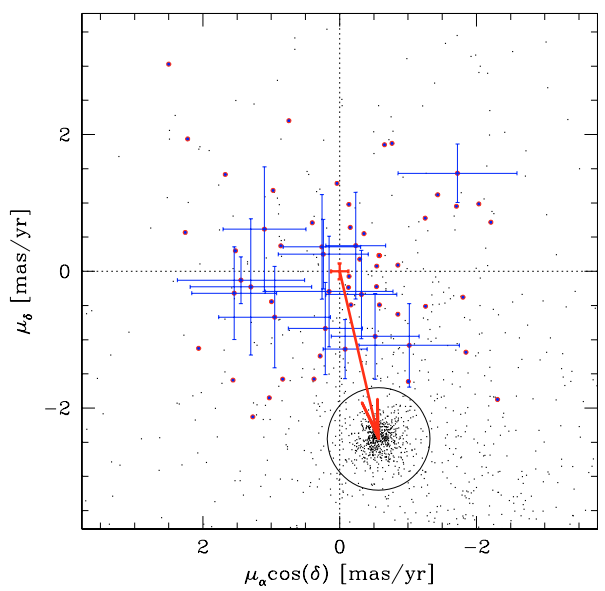

Fig. 1. Vector point diagram of the proper motions, in equatorial coordinates. Filled circles are the reference galaxies, and smaller dots are the stars.

of the stars. We then took a weighted mean, and found that this result depended almost completely on the $\sim 15$ galaxies whose errors are smallest.

Figure 1 shows the vector point diagram for the objects measured. Dots show the proper motions of the stars, and filled circles those of the galaxies. To avoid confusion, we show error bars for only the best 15 galaxies, each of which has an error $<1$ mas yr$^{-1}$ in each coordinate. The galaxies that have larger errors agree with the mean but contribute almost no weight to it. Cluster members form a tight clump below the middle of Fig. 1, within the $0.75-$ mas $\mathrm{yr}^{-1}$ circle that we have adopted to define cluster membership. (This radius was chosen as a compromise between losing cluster members and including field stars that have a motion close to that of the cluster stars.) The separation between field stars and cluster stars is well defined. The zero point of the figure was placed at the weighted mean motion of the galaxies, and is marked with dotted lines and with the error bars of the zero point. This zero-point determination is the dominant source of uncertainty in the absolute motion of NGC 6791.

With this zero point we find for NGC 6791 an absolute proper motion, in the $\mathrm{J} 2000.0$ system, of

$\left(\mu_{\alpha} \cos \delta, \mu_{\delta}\right)=(-0.57,-2.45) \pm(0.13,0.12) \mathrm{mas} \mathrm{yr}^{-1}$

These values represent a considerable change from the $\left(\mu_{\alpha} \cos \delta, \mu_{\delta}\right)=(-0.10,-0.70) \pm(0.90,0.80)$ mas $^{-1}$ used by Carraro et al. (2006). In Galactic coordinates (Fig. 2) our motions correspond to $\left(\mu_{\ell} \cos b, \mu_{b}\right)=(-2.45,-0.56) \pm$ $(0.13,0.13)$ mas $\mathrm{yr}^{-1}$. At the compressed scale of Fig. 2 many more field stars can be seen. The distribution of their proper motions is clearly elongated; this is the consequence of streaming effects due to differential rotation, for stars at different distances along the line of sight.

\section{Radial velocity}

For the third component of the motion of NGC 6791 (along the line of sight), we used the results in two recently published papers. In the first of these Gratton et al. (2006) obtained highresolution spectra of four red-clump stars; combining their measured radial velocities gives a mean value of $V_{\mathrm{rad}}=-47.2 \pm$ $1.5 \mathrm{~km} \mathrm{~s}^{-1}$. In the second paper, Carraro et al. (2006) derived a mean radial velocity $V_{\text {rad }}=-47.1 \pm 0.8 \mathrm{~km} \mathrm{~s}^{-1}$ from the spectra of 15 probable cluster members. We adopted as the radial 


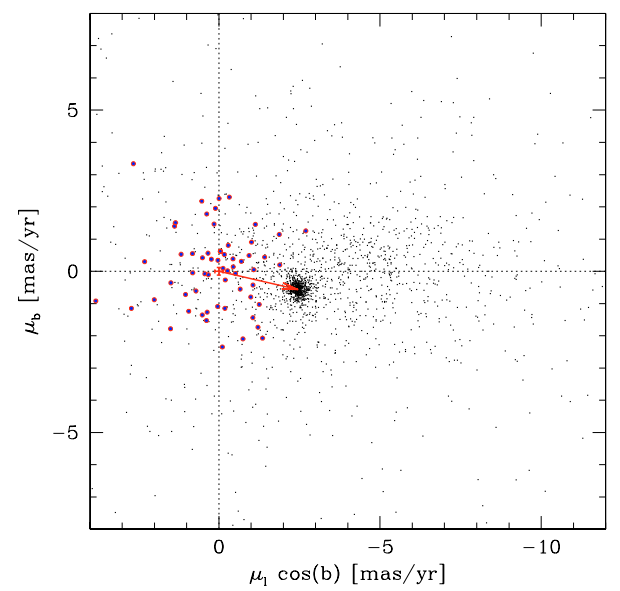

Fig. 2. Vector point diagram of the proper motions, in Galactic coordinates.

Table 2. Input conditions for orbit calculation. Distances are in $\mathrm{kpc}$, and velocities in $\mathrm{km} \mathrm{s}^{-1}$.

\begin{tabular}{cccccc}
\hline \hline$d$ & $U$ & $V$ & $W(=Z)$ & $\Pi$ & $\Theta$ \\
\hline 3.6 & $34 \pm 4$ & $-51 \pm 2$ & $-11 \pm 2$ & $40 \pm 4$ & $168 \pm 3$ \\
4.0 & $38 \pm 4$ & $-52 \pm 2$ & $-12 \pm 2$ & $43 \pm 4$ & $167 \pm 3$ \\
4.4 & $42 \pm 5$ & $-54 \pm 2$ & $-13 \pm 2$ & $47 \pm 4$ & $165 \pm 3$ \\
\hline
\end{tabular}

velocity of NGC 6791 the weighted mean of the two measurements, $V_{\text {rad }}=-47.1 \pm 0.7 \mathrm{~km} \mathrm{~s}^{-1}$.

\section{Calculation of the orbit}

Our absolute proper motion for NGC 6791, along with the radial velocity and an assumed distance of $4 \mathrm{kpc} \pm 10 \%$ (King et al. 2005), allows us to derive its three velocity components and calculate its Galactic orbit. The orbit should allow us to study the dynamical history of the cluster, and to assess the possible impact of the motion on its internal dynamics, its mass function, and its origin.

The integration of an orbit requires adopting a model of the potential of the Milky Way. We chose that of Allen \& Santillan (1991), which assumes a Galactocentric distance and rotation velocity for the Sun of $R_{0}=8.5 \mathrm{kpc}$ and $\Theta_{0}=220 \mathrm{~km} \mathrm{~s}^{-1}$, and takes densities in bulge, disk, and halo components whose combined gravitational force fits a rotation curve that agrees with observation. Besides being time-independent, their potential is axisymmetric, fully analytic, and mathematically very simple. It has already been used to derive the Galactic orbits of open clusters (Carraro \& Chiosi 1994; Carraro et al. 2006) and disk and halo globular clusters (Odenkirchen \& Brosche 1992; Milone et al. 2006). The potential is time-independent clearly a crude approximation, because a significant variation of the Galactic potential is expected over the lifetime of this cluster. Nevertheless, it is reasonable to believe that the real Galactic potential has not changed much in the last few Gyr, so that the parameters that we derive for the present-day orbit of NGC 6791, such as the apo- and perigalactic distances, can be considered to be reasonable estimates.

The initial conditions are given in Table 2 for three different heliocentric distances. The integration routine is a modified second-order Bulirsch-Stoer integrator (Press et al. 1992). The orbits were integrated back in time for $1 \mathrm{Gyr}$, and are shown in Fig. 3 both in the $x y$ plane (left panels), and in the meridional plane (right panels). The orbital parameters are given in
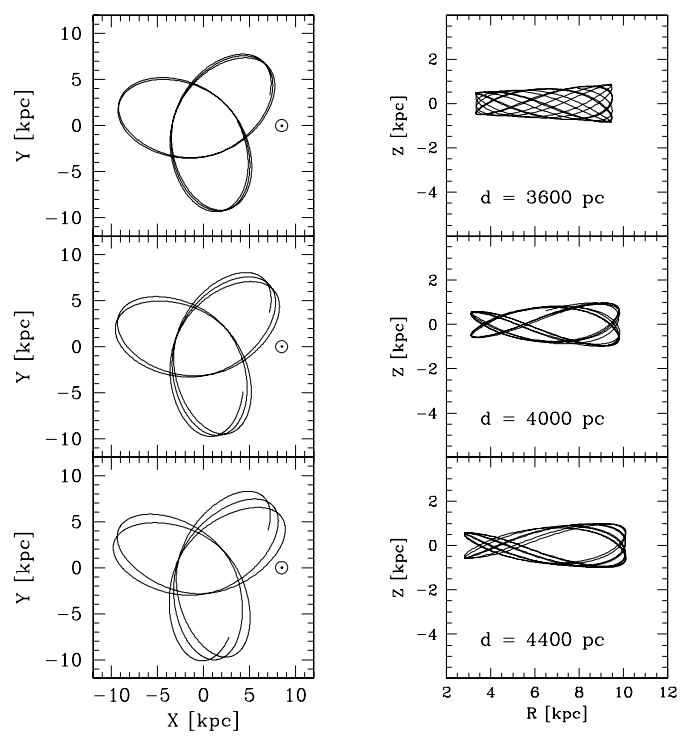

Fig. 3. Orbits calculated back in time for $1 \mathrm{Gyr}$, for assumed cluster distances of 3.6, 4.0, and $4.4 \mathrm{kpc}$.

Table 3. Orbit parameters, for the three different distances. Units: $d[\mathrm{kpc}], L_{z}\left[\mathrm{kpc} \mathrm{km} \mathrm{s}{ }^{-1}\right], E_{\mathrm{tot}}\left[10 \times \mathrm{km}^{2} \mathrm{~s}^{-2}\right], P[\mathrm{Myr}], R_{\mathrm{a}}[\mathrm{kpc}], R_{\mathrm{p}}[\mathrm{kpc}]$, $z_{\max }[\mathrm{kpc}], e$ [pure number].

\begin{tabular}{cccccccc}
\hline \hline$d$ & $L_{z}$ & $E_{\text {tot }}$ & $P$ & $R_{\mathrm{a}}$ & $R_{\mathrm{p}}$ & $z_{\max }$ & $e$ \\
\hline 3.6 & 1101 & -11085 & 120 & 9.50 & 3.32 & 0.86 & 0.48 \\
4.0 & 1060 & -11174 & 130 & 9.83 & 3.09 & 0.98 & 0.52 \\
4.4 & 994 & -11232 & 141 & 10.12 & 2.81 & 1.00 & 0.56 \\
\hline
\end{tabular}

Table 3, where successive columns give the assumed heliocentric distance of the cluster $(d)$, the $z$-component of the angular momentum $\left(L_{z}\right)$, the total energy $\left(E_{\text {tot }}\right)$, the orbital period $(P)$, the apo- $\left(R_{\mathrm{a}}\right)$ and pericenter $\left(R_{\mathrm{p}}\right)$ of the orbit, the maximum vertical distance the cluster reaches $\left(z_{\max }\right)$ and the eccentricity $(e)$, defined as $\left(R_{\mathrm{a}}-R_{\mathrm{p}}\right) /\left(R_{\mathrm{a}}+R_{\mathrm{p}}\right)$.

Our newly derived orbital parameters are based on a more accurate space velocity, and are thus an improvement over previous orbits. In particular, this is the first time that proper motions referred to extragalactic objects have been used in studying the orbit of NGC 6791.

The $\pm 10 \%$ range in distance does not change the shape of the orbit significantly, nor do the orbital parameters change greatly. The orbit is of boxy type. The eccentricity is significantly higher than is typical for an old open cluster. As the assumed heliocentric distance increases (from top to bottom panel) the cluster tends to have a longer period, to reach greater heights above the Galactic plane, and to show a larger epicyclic amplitude, dipping closer to the Galactic Center. The cluster never moves very far from the Sun toward the anticenter, while in the other direction it reaches rather small Galactocentric distances. Over one radial period it crosses the Galactic plane three times. (This is quite clear in the bottom panel on the right of Fig. 3; for other assumed distances it is less obvious, but equally true.)

Note that the new tangential motion derived in this paper leads to some sizable differences from previous results. The most important change is in the apogalactic distance $R_{\mathrm{a}}$, which in the present paper is strikingly smaller than previous values. This weakens significantly the likelihood of an extragalactic origin for NGC 6791, as proposed by Carraro et al. (2006).

In its life in relatively dense regions of the Milky Way, NGC 6791 has had a difficult time dynamically. In each orbital 
period of $\sim 130$ Myr it has endured a rapid Galactocentric passage at $R \sim 3 \mathrm{kpc}$, a disk crossing at $R \sim 9 \mathrm{kpc}$, and two more rapid crossings through the denser part of the disk at $R \sim 5 \mathrm{kpc}-$ all four of them producing tidal shocks. The survival of the cluster till the present era is probably due only to its high density and large mass. The mass has been decreasing with time, however. Internal equipartition keeps the lower-mass stars preferentially in the outer parts, and the tidal buffeting has detached much of low-mass population, leading to the flat mass function noted by King et al. (2005).

Another application of knowledge of the orbit of NGC 6791 is to the question of the origin of a super-metal-rich cluster that is $\sim 8 \mathrm{kpc}$ from the Galactic center. Grenon (1999) has suggested that the stars in the solar neighborhood that have comparably high metallicity could have originated in the Galactic bulge and then been perturbed by the central bar into orbits that bring them out to here. Our orbit suggests that a similar dynamical history might apply to NGC 6791.

\section{Summary and conclusions}

By identifying and measuring galaxies with point-like centers, we have been able to measure an absolute proper motion for NGC 6791. With the known radial velocity and the distance, we have computed the Galactic orbit of the cluster. Uncertainties in the orbit are due mainly to the inaccuracy of the distance, but partly to the unknown gravitational influence that the central bar of the Galaxy may have had on the orbit of the cluster. It is quite plausible that the high metallicity of NGC 6791 is associated with an origin in the inner region of the Galaxy.

Acknowledgements. G.P. acknowledges support by MIUR under the program PRIN2003. I.R.K. and J.A. were supported by STScI grants GO-9815 and GO-10471.

\section{References}

Allen, C., \& Santillan, A. 1991, Rev. Mex. Astron. Astrofis., 22, 255 Anderson, J., \& King, I. R. 2000, PASP, 112, 1360

Anderson, J. 2002, The 2002 HST Calibration Workshop, ed. S. Arribas, A. Koekemoer, \& B. Whitmore (Baltimore: STScI), 13

Anderson, J. 2005, The 2005 HST Calibration Workshop, ed. A. M. Koekemoer, P. Goudfrooij, \& L. Dressel (Baltimore: STScI), 11

Anderson, J., \& King, I. R. 2006, HST ACS/ISR 2006-01

Bedin, L. R., Piotto, G., Anderson, J., \& King, I. R. 2003, AJ, 126, 247

Bedin, L. R., Salaris, M., Piotto, G., et al. 2005, ApJ, 624, L45

Carraro, G., \& Chiosi, C. 1994, A\&A, 288, 761

Carraro, G., Villanova, S., Demarque, P., et al. 2006, ApJ, 643, 115

Gratton, R., Bragaglia, A., Carretta, E., \& Tosi, M. 2006, ApJ, 642, 462

Grenon, M. 1999, Ap. Sp. Sci., 265, 331

King, I. R., Bedin, L. R., Piotto, G., Cassisi, S., \& Anderson, J. 2005, AJ, 130, 626

Milone, A., Villanova, S., Bedin, L. et al. 2006, A\&A, 456, 517

Press, W. H., Teukolsky, S. A., Vetterling, W. T., \& Flannery, B. P. 1992, Numerical Recipes (Cambridge: Cambridge University Press), 718

Odenkirchen, M., \& Brosche, P. 1992, Astron. Nachr., 313, 69 